\title{
¿Realmente mató la bacteria al coronel? \\ Perspectiva sistémica, causación internivélica e intervalos de cuasi-descomponibilidad en las explicaciones mecanísticas*
}

\author{
(Did the bacterium really kill the colonel? \\ Systemic view, inter-level causation, and levels of quasi-decompositionality in mechanistic \\ explanations)
}

\author{
Emilio Cáceres Vázquez y Cristian Saborido
}

Recibido: $03 / 11 / 2017$

Versión final: 23/01/2018

BIBLID 0495-4548(2018)33:1p.129-148

DOI: $10.1387 /$ theoria.18295

RESUMEN: En este trabajo analizamos críticamente el enfoque mecanicista de explicación científica y proponemos complementar esta perspectiva con una concepción de nivel como intervalo de cuasi-descomponibilidad que nos permite fundamentar metafísicamente las propuestas mecanicistas clásicas en las propiedades sistémicas características de las entidades que se trata de explicar. A través del análisis de ejemplos concretos, demostraremos cómo nuestra propuesta permite superar algunas de las limitaciones de los enfoques predominantes de explicación mecanística.

Palabras clave: Mecanicismo, explicación científica, intervalos de cuasi-descomponibilidad, causalidad, perspectiva sistémica.

ABSTRACT: In this paper, we critically analyze the mechanicist approach to scientific explanation and propose to complement this view with a conception of level as interval of quasi-decompositionality. This conception allows us to metaphysically base the mechanicist proposals on characteristic systemic features. By means of concrete examples, we claim that this proposal is able to overcome some of the alleged limitations of predominant models of mechanistic explanation.

Keywords: Mechanicism, scientific explanation, intervals of quasi-decompositionality, causality, systemic view.

* Queremos agradecer las críticas y sugerencias aportadas por dos revisores anónimos de THEORIA, así como a la audiencia del II Seminario de investigación en nuevas corrientes en filosofía de la biología y de la medicina, celebrado en la UNED, Madrid, el 27 de mayo de 2017. Este trabajo se enmarca en el proyecto de investigación «Mecanismos en las ciencias: de lo biológico a lo social» (FFI2017-89639-P). 


\section{Introducción}

El concepto de mecanismo es clave para comprender ontológicamente y explicativamente los fenómenos observables (Kuhlmann y Glennan 2014, 337). Esto es algo especialmente patente en las posturas actuales de campos de la filosofía de la ciencia, como la filosofía de la biología, la filosofía de la tecnología o la filosofía de las ciencias sociales. Los principales postulantes de las explicaciones mecanicistas afirman que en ámbitos científicos se considera «hacer ciencia» al hecho descubrir y describir mecanismos (Machamer et al. 2000, 2) y, aunque siempre hay críticos a esta postura (véase Dupré 2013), hay un sólido consenso acerca de que la explicación por mecanismos es la mejor forma de explicación científica (Levy 2013, 100).

No obstante, es importante señalar que no hay en el debate filosófico actual una definición única de mecanismo, sino varias descripciones distintas de lo que, en principio, parecía una intuición clara para los científicos. Esta intuición podría expresarse diciendo que un mecanismo sería, en su sentido más general, un conjunto de partes que interaccionan entre ellas para dar lugar a un fenómeno. Cuál es la naturaleza de estas partes, cómo se asocian para formar un conjunto o qué tipo de interacción establecen entre sí son cuestiones abiertas que admiten tantas interpretaciones como postulantes.

En este trabajo analizaremos estas cuestiones y propondremos un nuevo enfoque mecanístico para abordarlas. En primer lugar, se ofrecerá un análisis crítico de la formulación más influyente del mecanicismo actual, la propuesta original de Machamer, Craver y Darden (Machamer et al. 2000), así como del posterior desarrollo llevado a cabo principalmente por Craver (2006 y 2007). Tras esto, se mostrará cómo esta formulación puede complementarse con una nueva propuesta que interpreta los niveles de un sistema como intervalos de cuasi-descomponibilidad (Cáceres y Saborido 2017) y que tiene implicaciones para la interpretación de las nociones de mecanismo y de causación internivélica. A lo largo de este trabajo, desarrollaremos algunos ejemplos concretos de explicación de fenómenos biológicos ${ }^{1}$, a fin de ver cómo esta propuesta puede servir al mecanicismo para dar respuesta a algunos de sus aspectos más problemáticos.

\section{El enfoque mecanicista: partes, actividades e interacciones}

Probablemente, la propuesta más significativa dentro del mecanicismo sea la desarrollada en el artículo germinal del denominado Nuevo Mecanicismo: Thinking about Mechanisms, de Peter Machamer, Lindley Darden y Carl F. Craver ${ }^{2}$ y en los trabajos posteriores de Craver.

1 Como bien nos ha señalado un revisor anónimo de THEORIA, los fenómenos biológicos tienen características diferenciadoras de los no biológicos, muchos de los cuales son también objeto de explicaciones mecanísticas. Sin embargo, son los ejemplos biológicos los que predominan ampliamente en los trabajos que conforman el actual mecanicismo (véase Machamer et al. 2000), (Bechtel y Abrahamsen 2005) o (Wright 2012), entre muchos otros). En todo caso, aunque los casos concretos que se exponen en este trabajo pertenecen principalmente al ámbito biológico, creemos que la propuesta que ofrecemos es válida para una reformulación general de las explicaciones mecanísticas.

2 En adelante denominaremos a esta propuesta MDC. Aunque existen otras propuestas de carácter similar pero con diferencias considerables, como las de Stuart Glennan (Glennan 1996, 2002) o William Bechtel (Bechtel y Abrahamsen 2005) (Bechtel y Richardson 1993), es en MDC en donde se presentan las líneas principales que conformarán el marco explicativo mecanicista desde entonces. 
Para MDC un mecanismo estaría formado por entidades y actividades organizadas que son productoras de cambio (Machamer et al. 2000, 3). Esta definición general es adoptada por todas las posturas mecanicistas. Glennan afirma que un mecanismo subyacente a un comportamiento es un sistema complejo que produce tal comportamiento por interacción de un número de partes de acuerdo con leyes causales directas (Glennan 1996, 52). Bechtel propone que un mecanismo es una estructura que desempeña una función en virtud de sus componentes, operaciones componentes y su organización. El funcionamiento orquestado del mecanismo es responsable de uno o más fenómenos (Bechtel y Abrahamsen 2005, 423-24).

Por lo tanto, un presupuesto básico del mecanicismo es la postulación de la existencia de partes discretas, es decir, objetos reales como moléculas o células en los cuales es posible descomponer un sistema más o menos complejo. Los mecanicistas consideran que para poder identificar partes genuinas de un sistema basta con encontrar entidades componentes del mismo que tengan unas características estables, sean físicamente detectables mediante diferentes métodos, se puedan usar para intervenir en otros procesos, sean fisiológicamente plausibles y sean relevantes para explicar el fenómeno (Craver 2006, 371).

Un segundo aspecto a tener en cuenta es que, además de considerar que las partes son entidades reales y diferenciadas, MDC da la misma consideración ontológica a las actividades, adoptando de este modo una postura claramente dualista frente a posiciones sustantivistas como las de Bechtel (Bechtel y Richardson 1993) o Cartwright (Cartwright 1994) o de los ontologistas de procesos como Rescher (Rescher 1996)3. Para MDC un sistema puede descomponerse en partes y actividades, siendo las actividades lo que las partes hacen y lo que las engarza entre sí conformando un mecanismo. En otras palabras, es la relación entre partes establecida a través de sus actividades lo que conforma la organización de un sistema, y esta organización es explicable en términos mecanísticos.

El tercer aspecto que emana de la definición de mecanismo es el referido al tipo de interacción entre las entidades. Las explicaciones en términos de mecanismos hacen referencia a la relación causal entre las partes y sus actividades. Aunque no hay un consenso claro entre los mecanicistas acerca de cómo debe entenderse la causalidad ${ }^{4}$, puede defenderse que el enfoque causal del mecanicismo parte del enfoque de Wesley Salmon (Salmon 1994) y Phil Dowe (Dowe 2000) de las cantidades conservadas y de la perspectiva manipulacionista de James Woodward (Woodward 2003) y su interacción etiológica ideal.

El cuarto y último aspecto general del mecanicismo hace referencia también a la organización, concretamente a la estructura jerárquica presente en muchos de los sistemas estudia-

3 Stuart Glennan, otro filósofo del neo-mecanicismo, contrasta la caracterización ontológica de las actividades de MDC con su propio enfoque (Glennan 1996) y el de Bechtel y Richardson (1993) claramente sustantivistas. Kuhlmann y Glennan apuntan $(2014,340)$ que este dualismo podría tener sentido para aclarar que no se puede hacer referencia solo a las partes, a pesar de sugerir una independencia implausible entre entidades y actividades. Aun así, le resta importancia por ser una idea no desarrollada posteriormente, a pesar de seguir siendo la base de la definición de mecanismo en los trabajos más recientes de Craver $(2007,64,171,177,189)$, (Craver 2013, 7).

4 El desacuerdo emana del tratamiento óntico concreto de las actividades que se plantea MDC. Aunque autores como Kuhlmann y Glennan $(2014,340)$ tratan de minimizar la importancia de este desacuerdo, autores como Craver $(2007,64,171,189)$ han insistido en el mismo reivindicando sus implicaciones para la interpretación de la validez y el alcance del mecanicismo. 
dos por los científicos, que permite identificar diferentes niveles de organización de la materia y es fundamental en las actividades llevadas a cabo por él. La clave es que cada elemento componente de un mecanismo puede ser a su vez otro mecanismo y, por lo tanto, estaría igualmente formado por elementos organizados que interaccionan entre sí de forma causal directa. Consecuentemente, sería posible descomponer un mecanismo en otros mecanismos, lo que introduce en el marco mecanicista la idea de nivel.

Los mecanicistas insisten en resaltar que los mecanismos son, sobre todo, herramientas conceptuales que han de servir para la explicación de los fenómenos y, por lo tanto, han de permitirnos abordar características del fenómeno a explicar. Cuando los científicos apelan a mecanismos están siempre eligiendo un nivel concreto de organización. Es decir, dentro de la jerarquía anidada de un sistema, se escoge el nivel más pertinente para abordar el fenómeno que se quiere explicar y se apela a los mecanismos, sus elementos y sus relaciones causales que se localizan a este nivel. En la siguiente sección, abordaremos con mayor detalle esta idea de nivel y sus implicaciones para el marco mecanicista.

\section{La idea de nivel en el mecanicismo y las explicaciones de los fenómenos biológicos}

\subsection{LOS NiVELES DE NATURALEZA}

Un aspecto clave para poder definir un mecanismo es el criterio que se adopta para la elección de las entidades concretas en las que poder descomponer un sistema y, consecuentemente, el concepto de nivel de organización de la materia que este criterio implica.

Craver distingue entre elementos de ciencia y elementos de naturaleza (Craver 2007, 171). Dentro de los elementos de ciencia (análisis, descripciones, teorías) sitúa los niveles clásicos que Oppenheim y Putnam establecieron en 1958 y la versión «afinada» de Wimsatt $(1976,253)$, esto es, partículas elementales, átomos, moléculas, células, seres vivos multicelulares y grupos sociales (Oppenheim y Putnam 1958, 9).

En los niveles de naturaleza, Craver agrupa aquellos elementos que describen ítems del mundo (actividades, entidades, propiedades y estados), y los distingue en tres clases: causales, de tamaño y de composición. Los niveles causales hacen referencia a relaciones de causa y efecto entre elementos, como la regulación del metabolismo. Los niveles de tamaño apelan a la dimensionalidad de los componentes, tal y como hace, por ejemplo, el modelo de Churchland y Sejnowski (1988, 741-42). El tercer y último grupo es el de niveles de composición, que a su vez se va a dividir en cuatro subtipos: mereológico, de agregatividad, de mera contención espacial y de mecanismo. El primero de ellos sería el enfoque más reduccionista, pues implica la inexistencia de propiedades emergentes. Craver descarta su validez explicativa en campos en los que su asunción de la existencia de propiedades emergentes es indiscutible, como la neurobiología ${ }^{5}$. En segundo lugar, la referencia de Craver a niveles de agregatividad, esto es, niveles en los que sus componentes no tienen ninguna relación entre ellos más que los de acumular cantidad, se basa principalmente en las condiciones expuestas por Wimsatt, esto es, intersustitución, escala de tamaño, descomposición y reagregación y linealidad (Wimsatt 1997, S376). En tercer lugar, identifica lo que denomina niveles de

5 La asunción del principio emergentista clásico «[...] los mecanismos son siempre literalmente más que la suma de sus partes» (Craver 2007, 185-86) es significativa del enfoque del autor. 
mera contención material/espacial para referirse a la división de un todo, más que en partes que actúan de forma interrelacionada, en particiones arbitrarias, como sería la división de un puzle en piezas. Esta división resulta inútil desde una perspectiva explicativa, pero le sirve para poner de manifiesto la necesidad de un análisis top-down previo a la «decisión» de una división con sentido explicativo. Por último, Craver se centra en los niveles de mecanismos, la división más pertinente desde un punto de vista científico y explicativo y que va a defender como la base de la explicación mecanicista.

La explicación por mecanismos se utiliza de forma habitual dentro de la biología para la elucidación de la forma en la que transcurren las interacciones entre moléculas y agrupaciones de éstas, especialmente dentro del campo de la biología molecular y de la fisiología celular. De hecho, muchos de los ejemplos a los que aluden los mecanicistas son ejemplos de mecanismos biológicos, como el mecanismo de la visión o el de la sinapsis neuronal ${ }^{6}$.

Uno de los ámbitos preferidos por los mecanicistas y especialmente por Craver es el de la neurobiología. En su análisis de cómo funcionan las explicaciones neurobiológicas, Craver ve una defensa clara de su propuesta mecanicista. Así, considerando las características propias del sistema neuronal, Craver afirma que la mejor opción para dar cuenta de los fenómenos neurológicos es la de descomponer el sistema neuronal en niveles de composición, pero no con base simplemente espacial o material, sino en niveles que supongan una identificación de mecanismos concretos (Craver 2007, 189).

Estos niveles mecanísticos estarían formados por entidades y actividades que cumplen dos condiciones. Por una parte son composicionales, pero no simples niveles de tamaño que pueden alojar regularidades. Por otra, Craver destaca que estos niveles no están formados por objetos pasivos correspondientes a entidades clásicas, sino por objetos actores. Considerando una sinapsis neuronal, una división clásica distinguiría dos neuronas, entendiendo la brecha interneuronal como una separación entre componentes, mientras que el enfoque mecanístico entiende la porción terminal de la neurona pre-sináptica, la hendidura y la porción inicial de la neurona post-sináptica como un mecanismo. Resulta así evidente que el mecanicismo supone una visión enfocada a la función (véase Garson 2013). Craver cree que es mejor hablar de jerarquías particulares en vez de estructuras anidadas generales pues, según su propuesta, solo puede decirse que dos objetos forman parte del mismo nivel si son componentes del mismo mecanismo, es decir, si contribuyen a la misma función (Craver 2007, 192-93), (véase, también, Craver 2001).

\subsection{LA CAUSACIÓN INTERNIVÉLICA}

Un mecanismo se puede descomponer en componentes que se consideran del mismo nivel, pero al descomponerlos de nuevo nos encontramos con la postulación de nuevos niveles. Una de las cuestiones centrales del mecanicismo es clarificar si se da una relación causal no solo entre elementos de un mismo nivel a través de un mecanismo, sino también entre me-

6 Además de esta abrumadora presencia de ejemplos biológicos en los trabajos mecanicistas, en muchos de estos trabajos también se han presentado casos relativos a otros ámbitos, como los ejemplos de mecanismos en el mundo cuántico (Kuhlmann y Glennan 2014) y a nivel poblacional y social (Hedström y Ylikoski 2010). En este artículo nos centraremos en los casos biológicos, sin pensar por ello que las observaciones que aportamos no puedan ser extrapoladas a las explicaciones basadas en mecanismos para otras ciencias (véase Steel 2007). 
canismos que son localizables en diferentes niveles. Craver y Bechtel (2006) han abordado esta problemática cuestión de la causación internivélica y han propuesto una solución a la misma que se basa en tres puntos (Craver y Bechtel 2006, 550).

Por una parte, Craver y Bechtel distinguen la cuestión de la causación internivel de la relación causal entre entidades de diferente tamaño. Según lo explicado arriba, las relaciones causales entre las partes se establecen por su pertenencia a un mismo mecanismo y los componentes de un mismo mecanismo pertenecen consecuentemente a un mismo nivel, por lo que hablar de relación internivel entre mecanismos diferentes o entre componentes de esos mecanismos carece de sentido. La única relación posible entre niveles es de constitución, es decir, mecanismos y elementos de un nivel más bajo componen los mecanismos de niveles más altos.

En segundo lugar, Craver y Bechtel afirman que los niveles están definidos contextualmente, lo mismo que las ciencias, insistiendo en que no hay razón por la que una categoría ontológica, como las moléculas, no pueda interaccionar con otra, como las células, aun cuando su categorización se haya hecho siguiendo criterios distintos.

Para finalizar, Craver y Bechtel intentan compatibilizar su enfoque mecanístico con la emergencia de propiedades. Para ellos, los niveles superiores son siempre explicables en términos de mecanismos. En contraposición, consideran que solamente sería fuertemente emergente aquel fenómeno que no puede explicarse por mecanismos, incluso aunque emerja de la organización de un mecanismo (Craver y Bechtel 2006, 551). Sin embargo, afirman que lo fuertemente emergente emana de una consideración de nivel caracterizada por no ser de tipo constitutivo, y que por lo tanto no se correspondería con la concepción de nivel por ellos defendida. Además, afirman que la idea de la causación internivel con respecto al surgimiento de propiedades emergentes es diferente de la causación descendente en propiedades mundanas, por lo que creen que pueden desvincular la idea de emergencia de la de causación descendente (Craver y Bechtel 2006, 550). Craver y Bechtel consideran que esta interpretación de la relación internivélica se corresponde con el pensamiento científico. Afirman explícitamente que la forma de trabajar de los científicos tiene mucho que ver con la consideración de la causalidad internivel, pues estos diseñan los experimentos buscando la correlación entre micro-estructuras y macro-funciones (Craver y Bechtel 2006, 553).

El enfoque mecanicista ha sido profusamente criticado por distintas razones por autores como Barberis (2012), Levy (2013) o Wright (2012), entre otros. En este trabajo no se pretende hacer una nueva crítica a este modelo explicativo, sino tratar de fortalecerlo contrastando dicho enfoque con nuestro trabajo previo (Cáceres y Saborido 2017), ofreciendo una alternativa sólida a las formulaciones predominantes del mecanicismo.

El mecanicismo en sus formulaciones predominantes tiene una ambición claramente epistemológica o explicativa (Levy 2013,100), y por ello sacrifica la adecuación óntica en favor de la comprensión. Tal y como puede verse en los trabajos de Craver, la definición de niveles de mecanismo está basada más en la pertinencia explicativa con respecto a los intereses del investigador que en las propiedades organizacionales de los sistemas cuyos fenómenos se pretende explicar. Sin embargo, cabe preguntarse si un modelo explicativo adecuado para las ciencias biológicas no debería realmente partir de la concreción de la naturaleza de la materia biológica, por lo que una adecuada definición de nivel que tenga en cuenta las propiedades distintivas de los sistemas biológicos sería clave para el correcto desarrollo de una idea de mecanismo apropiada para la biología.

En la siguiente sección mostraremos cómo el enfoque mecanicista puede verse enriquecido si se cuenta con un criterio para la compartimentación de la materia de forma que de- 
fina niveles y entidades y que, a partir de ahí, permita desarrollar un modelo explicativo basado en entidades que se comportan como mecanosistemas ${ }^{7}$.

\section{Mecanosistemas, intervalos de cuasi-descomponibilidad y explicación mecanicista}

\subsection{INTERVALOS DE CUASI-DESCOMPONIBILIDAD COMO NIVELES DE ORGANIZACIÓN}

En sus pioneros trabajos en teoría de la complejidad, Herbert Simon distinguió dos tipos de sistemas en función de las relaciones entre sus subsistemas, denominando sistemas descomponibles a aquellos en los que las relaciones entre sus subsistemas eran claramente distinguibles y sistemas cuasi-descomponibles (《nearly-decomposable systems» en su terminología) a los que dichas relaciones eran no tan claramente discernibles si bien no despreciables (Simon 1962, 473). Estos últimos se caracterizan en que a corto plazo el comportamiento de sus subsistemas es aproximadamente independiente entre sí, mientras que a largo plazo dependen solo de forma agregativa.

Para ilustrar esta distinción, Simon propuso un ejemplo basado en la existencia de un sistema aislado que podía descomponerse o no en subsistemas anidados (Simon 1962, $474)^{8}$. La clave del ejemplo de Simon, y de la comprensión de la dicotomía descomponible/ cuasi-descomponible, es la arbitrariedad de la elección. Simon hablaba de una casa perfectamente aislada dividida en habitaciones bien aisladas subdivididas a su vez en cubículos mal aislados en cuyo interior existía un termómetro. La clave de este ejemplo estaba en que, partiendo de una situación de desequilibrio térmico y pasado un tiempo, la uniformización de la temperatura iría haciendo innecesarios los termómetros, pudiendo eliminar primero los de los cubículos y después los de las habitaciones, bastando finalmente con uno que marcara la temperatura de toda la casa (Simon 1962, 474).

Como ya se señaló en (Cáceres y Saborido 2017, 95), el problema de este ejemplo radica en que es imposible extrapolarlo al mundo natural, pues en él nos hemos de enfrentar con la inexistencia de sistemas aislados. De hecho, ni siquiera el planeta Tierra es un sistema aislado que pueda considerarse como un sistema descomponible? ${ }^{9}$ Parece más bien que el mundo natural debe entenderse como un continuo material que podemos cuasi-dividir en componentes anidados en base a ciertos criterios de cuasi-descomponibilidad. Paul Weiss lo expresaba así:

Para mí, como observador de la naturaleza, el universo se presenta a modo de un inmenso continuo cohesivo. Sin embargo, por lo común no lo vemos así. Estamos acostumbrados a mirarlo

7 Para no dar lugar a confusiones y colaborar a la proliferación de más interpretaciones de la palabra mecanismo (Levy 2013), en este trabajo se va a utilizar el término mecanosistema, ya introducido en (Cáceres y Saborido 2017). Además, tal y como se explicará, este término de mecanosistema nos ayudará a remarcar las diferencias entre nuestra concepción de mecanismo de la propuesta por MDC y otros autores afines.

8 Este ejemplo se analiza detalladamente en (Cáceres y Saborido 2017, 94).

9 Esto también afecta al criterio de descomponibilidad que proponen Bechtel y Richardson y en el que basan su propuesta mecanicista: «si el grado de integración entre los componentes de un todo no anula a los componentes como entidades autónomas, el resultado es una jerarquía parte-todo descomponible» (Bechtel y Richardson 1993, 28). Quizá sería una propuesta teóricamente válida para un criterio de cuasi-descomponibilidad, pero no de descomponibilidad. 
como un conglomerado de fragmentos regulares. Este hábito deriva en parte de la herencia biológica, que lleva a enfocar las cosas, tales como presas, enemigos u obstáculos, como necesidad vital; en parte procede de la tradición cultural, y también de la curiosidad, que dirige nuestra atención e interés hacia «objetos» limitados. Éstos pueden consistir en conjuntos bien delimitados en nuestro campo visual, en series repetidas de sonidos en el canto de los pájaros, la música o la voz humana, o en procesos de modelo regular, como las ondas. Lo que atrae nuestra atención es su aparición reiterada en forma constante y duradera: al menos se mantiene lo suficiente o se repiten en nuestra experiencia lo bastante a menudo para merecer un nombre, en contraposición a las constelaciones que les rodean, mucho menos regulares y más fugaces, a las cuales, en contraste, llamamos «fondo». (Weiss 1970,7)

Simon introdujo en su propuesta la consideración de un valor $\varepsilon$ que decidimos arbitrariamente y que nos sirve como delimitador ${ }^{10}$. Es evidente que si cambiamos el valor de $\varepsilon$ o criterio de cuasi-descomponibilidad podríamos afirmar que uno u otro subsistema es cuasidescomponible. De igual manera, si en vez de utilizar un solo criterio, usamos dos, $\varepsilon_{1}$ y $\varepsilon_{2}$, tendremos la distinción entre dos subsistemas cuasi-descomponibles anidados.

Siguiendo con el ejemplo de Simon, podríamos elegir diferentes $\varepsilon_{n}$ según el subsistema al que nos queramos referir, que nos diferenciarían cubículos, habitaciones y casas. Así tendríamos que cada subsistema cuasi-descomponible estaría delimitado por dos $\varepsilon_{n}$ y sería definible por lo que podríamos llamar intervalos de cuasi-descomponibilidad ${ }^{11}, I_{(i, j)}^{n}=\left[\varepsilon_{\mathrm{i}}, \varepsilon_{\mathrm{j}}\right]$. Con esta nomenclatura, tendremos cubículos o $I_{(0,1)}=\left[\varepsilon_{0}, \varepsilon_{1}\right]$, habitaciones o $I_{(1,2)}=\left[\varepsilon_{1}, \varepsilon_{2}\right]$ y casas o $I_{(2,3)}=\left[\varepsilon_{2}, \varepsilon_{3}\right]$. Todos serían sistemas cuasi-descomponibles y por tanto se influyen de forma no despreciable.

Otra característica notable del sistema de Simon es que evoluciona hacia un equilibrio, algo que difícilmente ocurrirá mientras existan generadores de desigualdad. En el ejemplo de la casa ocurrirá si introducimos radiadores en los cubículos. En este caso se establecería un equilibrio dinámico de manera que habrá un gradiente térmico centrado en los radiadores $^{12}$. Por lo tanto, dentro de esta diferencia, la decisión de instalar un solo termómetro, bien en una habitación, bien en una vivienda, etc, es una decisión arbitraria dependiente del interés del observador. Resulta evidente que esta decisión tendrá como consecuencia la pixelación de la temperatura, con la pérdida de información que esto supone. Dependiendo del foco del análisis, el píxel será más o menos grande, provocando una uniformización de la temperatura y un efecto de parcelación del continuo material. La decisión puede ser útil, pero no inocua, y las consecuencias pueden ser más o menos importantes, pero no nulas. En el caso real del control térmico de una casa, la práctica de situar un solo termostato tiene la consecuencia de crear un gradiente térmico en el interior de la casa. El único lugar en el que podemos asegurar la temperatura programada es justo donde esté situado el termostato.

Podrá resultar útil considerar que la vivienda es un píxel de una temperatura concreta pero es evidente que no es real. Vale la pena hacer el esfuerzo de trasladar este ejemplo de Si-

10 Es interesante señalar que este valor podría coincidir con la autonomía de Bechtel y Richardson (1993, nota 9).

11 En adelante ICD.

12 En un sistema aislado, cuando existe un generador de desigualdad se llegaría a una situación de equilibrio térmico al alcanzar la casa la temperatura de los radiadores. Si el sistema fuera cuasi-descomponible la vivienda tendría pérdidas y no moriría por calor, formando un sistema alejado del equilibrio. Gracias a Daniel Ruiz-Castillo por esta observación. 
mon al mundo real, es decir, considerar cómo se comporta la temperatura en casas reales. Si analizamos la casa desde el exterior y ampliamos aún más el foco, a una ciudad por ejemplo, vemos que la influencia de cada cubículo en muy pequeña, pero no es despreciable, pues aunque es una cantidad mínima comparada con la procedente del sol, es la responsable de que la temperatura en los pueblos sea sensiblemente mayor que en una zona deshabitada. Por lo tanto, la segunda condición de Simon para los sistemas cuasi-descomponibles, «a largo plazo, el comportamiento de cualquiera de los componentes depende únicamente de modo agregativo del comportamiento del resto de los componentes» (Simon 1962, 474), no se satisface cuando observamos una versión realista de su ejemplo paradigmático, pues en este caso los sistemas siguen generando desigualdad.

Consciente de que este era el caso también para los sistemas naturales, Simon propuso (Simon 1962, 475) aplicar un criterio $\varepsilon$ basado en la diferencia energética entre enlaces covalentes y débiles para determinar la (des)composición de la materia. De esta manera, en función del valor de $\varepsilon$ elegido, podemos parcelar el continuo material. Para un valor de $\varepsilon$ justo por debajo del valor energético del enlace covalente, estableceremos que las moléculas son sistemas cuasi-descomponibles, mientras que aquellas uniones basadas en enlaces más débiles, como los enlaces por puente de hidrógeno, son interacciones intermoleculares. Consecuentemente, consideramos el $\mathrm{H}_{2} \mathrm{O}$ como una molécula, mientras que la interacción entre varios hidrógenos y oxígenos por enlaces covalentes y de hidrógeno $\left(\mathrm{H}_{2} \mathrm{O}\right)_{n}$ se ven comúnmente como simples conjuntos de moléculas y no como macromoléculas.

Partiendo de esta idea, podemos dividir la materia en niveles en función de nuestra elección del criterio de cuasi-descomponibilidad. Así, en función de $\varepsilon_{n}$ habrá un «nivel fundamental aproblemático» en términos de (Machamer et al. 2000, 13) o microscópico $F^{13}$, un nivel macroscópico $M$ y múltiples niveles mesoscópicos anidados $m_{i}, m_{j}, \ldots m_{n}$.

Comenzando en los átomos ${ }^{14}$, éstos tienen ciertas propiedades debidas a su composición cuantitativa que determinan la forma en la que combinan entre si. Una de ellas, predominante en la materia viva, es la unión covalente ${ }^{15}$ que forma las moléculas. A esta escala, esta unión es lo suficientemente estable como para considerar la energía necesaria para romperlos un nuevo criterio de cuasi-descomponibilidad y, a fin de cuentas, un nuevo nivel, el molecular. A partir de aquí, las relaciones entre moléculas, fuerzas intermoleculares, ión-ión, ión-dipolo, ión-dipolo inducido, hidrofóbicas y de van der Waals, conforman un nuevo plano. La interacción hidrofóbica entre ciertos lípidos da lugar a la membrana plas-

13 Por nivel F se entiende el nivel de las partículas fundamentales descritas por la Física, nivel que es considerado habitualmente como el nivel último que constituye la base para un reduccionismo ontológico. Actualmente el Modelo Estándar de Partículas considera que existen dos tipos de partículas, leptones y quarks, que carecen de estructura interna, por lo que se consideran partículas verdaderamente fundamentales. Además de MDC, quienes consideraban este nivel como «aproblemático», también Glennan $(1996,50)$ hizo referencia a este nivel fundamental y ha trabajado en la compatibilización del nivel cuántico con las explicaciones mecanísticas (Kuhlmann y Glennan 2014). Desde la Física, Roger Penrose (1991, 374-75) aclara la diferencia entre el nivel cuántico y el clásico, señalando explícitamente que las dificultades de la Física actual no suponen realmente un reto para el enfoque reduccionista.

14 Por economía de espacio, comenzamos la exposición en el nivel atómico, pero igualmente podríamos partir de leptones y quarks y, pasando por hadrones, llegar a los átomos. Las fuerzas nuclear fuerte, residual y débil serían nuestros $\varepsilon_{n}$.

15 Las uniones iónicas y metálicas son igualmente explicables en función de sus propiedades. 
mática, «frontera» que marca los límites de las células presentes en todos los sistemas vivos. Consecuentemente, la fuerza de las interacciones hidrofóbicas puede resultar un buen criterio de cuasi-descomponibilidad que delimite un nuevo nivel. Pero ahora el límite del ICD determina un borde que contiene en su interior una gran cantidad de moléculas de diferente naturaleza que interaccionan gracias a las fuerzas anteriormente citadas. Dentro de las células existen compartimentaciones basadas en membranas, los orgánulos, pero debido a que no existen en todos los tipos de células es preferible usarlo como un subnivel.

Escalando niveles, las células se unen (uniones estrechas, de adherencia, desmosomas, hemidesmosomas o uniones gap) dando lugar a diferentes estructuras, bien de células similares, bien diferentes, forman agrupaciones que cuentan con una barrera delimitadora. Esta barrera podría ser un nuevo criterio de cuasi-descomponibilidad y determinar el nivel de organismo. Un nivel formado por individuos del mismo tipo, el nivel de población, y uno superior formado por individuos de diferente tipo, incluyendo materia inerte, daría lugar al nivel de ecosistema, al de bioma y al de biosfera. Entre niveles se podrían establecer las divisiones clásicas, como tejidos, órganos, aparatos, entre el nivel de organismo y el celular u orgánulos y macromoléculas, entre el celular y el molecular, con el fin de afinar el nivel de estudio.

De esta partición longitudinal de la materia se sigue que la relación entre los subsistemas superiores y los inferiores es simplemente composicional y, por lo tanto, la relación entre dos niveles diferentes es de identidad. Por tanto, un bioma es un conjunto de ecosistemas, un ecosistema un conjunto de poblaciones, una población un conjunto de individuos, un individuo un conjunto de células, una célula un conjunto de moléculas, una molécula un conjunto de átomos y un átomo un conjunto de partículas elementales. El individuo es un punto intermedio de un conjunto de sistemas cuasi-descomponibles anidados.

Ahora bien, ¿cómo decidimos cuál es el subsistema relevante para la investigación? Resulta evidente que la elección de estos $\varepsilon_{\mathrm{i}}$ implica un sesgo observacional basado en un análisis top-down previo. Podríamos eliminar, o cuando menos minimizar, este sesgo estableciendo un nivel arbitrario pero sin observación previa proponiendo, por ejemplo, un nivel cada ciertas unidades de intensidad de interacción, pero esto conduciría a una división probablemente muy poco útil, equiparable a la de los niveles de mera contención material/espacial de Craver $(2007,187-88)$.

Una vez definidos los ICD, la disciplina encargada de su estudio debe realizar una descripción del objeto que pretende explicar acorde a lo observable desde ese nivel, demarcando así los límites del sistema que va a considerar (véase Cáceres y Saborido 2017, 97). Cada ciencia estudiará su propio intervalo y se encargará de describirlo estructural y funcionalmente ${ }^{16}$, distinguiéndolo así de su entorno y del resto de intervalos. Esta descripción debe hacerse en función de la metodología de la ciencia en cuestión e implica normalmente una terminología propia. Es decir, cada ciencia se centra en las propiedades detectables

16 Esta distinción entre los aspectos estructurales y los funcionales merece un tratamiento teórico independiente. Efectivamente, ciertos sistemas, tales como los biológicos, muestran características diferenciadoras que van más allá de las meramente estructurales (agradecemos a un revisor anónimo de THEORIA el que nos señalase este punto). Aunque esto supera los objetivos de este trabajo, podemos adelantar que nuestra propuesta sería que las actividades biológicas, las funciones, emanan de la estructura y, por lo tanto, dependen en último término de esta. Cómo la noción de función biológica puede ser abordada desde la perspectiva de los ICD es el objeto de una ulterior investigación. 
desde su nivel mediante sus métodos de investigación ${ }^{17}$. Por lo tanto, tratará su intervalo tomando solo aquellos inputs y outputs que considere necesarios para la finalidad del estudio, estableciendo un sistema modelo y haciendo una simplificación heurística de la realidad por razones pragmáticas. Las leyes científicas que actúan a cada nivel serían las regularidades detectadas durante la descripción de los sistemas que podrán o no ser catalogadas como leyes.

Esta modelización implica una simplificación que se amplifica conforme ascendemos de nivel. Los píxeles determinados al elegir un nivel de observación pueden resultar suficientemente útiles desde cierta distancia, pero no es cuestionable el hecho de que restan resolución. Así, en el estudio que hace Craver de la liberación de neurotransmisores mediada por calcio (Craver 2007, 22), se toma en consideración la concentración de cationes calcio, pero no la de sodio y potasio, responsables de la despolarización-repolarización de membrana que producen la liberación de calcio. Esta selección de los elementos que forman el sistema introduce sesgos que alteran los resultados, lo que conlleva asumir que, a la hora de introducir generalizaciones, éstas serán igualmente inexactas ${ }^{18}$, aunque lo suficientemente aproximadas para ser prácticas y permitir la explicación y la predicción.

\subsection{ENTIDADES-SISTEMA CON PROPIEDADES}

Ya hemos señalado que la definición dual de mecanismo, que considera la existencia tanto de entidades como de actividades, es clave para el posterior desarrollo del modelo de MDC, y probablemente uno de sus puntos más susceptibles a la crítica. La idea de nivel como ICD implica que solo las entidades tienen adecuación óntica, además de la innecesaridad explicativa de considerar ónticamente a las actividades. Solo las entidades del nivel F tienen propiedades no descomponibles que se deben a su propia naturaleza, mientras que las entidades resultantes de la elección de un criterio de cuasi-descomponibilidad tendrán un conjunto de propiedades de nivel ${ }^{19}$ resultantes de la simplificación realizada. Así, tendremos entidades-sistema con propiedades de nivel. De esta manera se engloba el enfoque de entidad que Kuhlmann y Glennan consideran común al Nuevo Mecanicismo. Para ellos las entidades son partes reales, posicionándose en un punto de vista perspectivista en el que destacan el para qué del mecanismo (Kuhlmann y Glennan 2014, 339).

Si una entidad de un mecanismo es una parte real y relativamente estable (Glennan 2008, 378), no es sencillo justificar su descomposición en otras partes igualmente reales y relativamente estables ¿Qué criterio seguimos para descomponer un sistema? Como veremos en este apartado, el enfoque basado en la cuasi-descomponibilidad entiende las partes de un sistema solo como arbitrariamente estables y puede facilitar resolver el problema de la descomposición mecanística.

17 Esta división en niveles estaría catalogado en la taxonomía de Craver $(2007,171)$ como un nivel de ciencia, pues los niveles así descritos son evidentemente un constructo epistémico, de la misma forma que lo son el de Oppenheim y Putnam o el de Wimsatt. Sin embargo, los propios niveles de mecanismos propuestos por Craver son también del mismo tipo, pues las entidades y las actividades que constituyen sus mecanismos, especialmente estas últimas, son también construcciones teóricas.

18 Debido a esto se dan excepciones, fenómeno que Glennan achaca al entorno (Glennan 2008, 378).

19 Estas propiedades son consideradas tradicionalmente como propiedades emergentes, cfr. (Cáceres y Saborido 2017, 101) 
De esta forma, tendremos por tanto que los átomos son sistemas del nivel atómico con propiedades de nivel derivables de las de sus elementos constituyentes. Dentro de estas propiedades podemos observar, por ejemplo, la electronegatividad (tendencia a ganar o perder electrones que proporciona una mayor estabilidad) o el radio iónico (radio del átomo tras ganar o perder electrones). Así, el neón tendrá propiedades cuantitativamente diferentes de las del flúor y de las del sodio. De estas propiedades dependerá el comportamiento de un elemento. Como consecuencia, en función de su electronegatividad un átomo ganará electrones, y por tanto se reducirá, o los perderá, oxidándose. Así, el flúor tenderá a captarlos, el sodio a cederlos y el neón a quedarse como está. En una situación sin injerencias, el flúor no se oxidará ni el sodio se reducirá, por lo que no se puede desvincular la acción de la propiedad y por lo tanto de la entidad. Si siguiéramos el modelo teórico propuesto por MCD, parecería que no hay conexión entre actividades y entidades y tanto el flúor como el sodio podrían oxidarse o reducirse. A esta independencia implausible se refieren Kuhlmann y Glennan cuando tildan de escalofriante este dualismo (Kuhlmann y Glennan 2014, 340).

Relacionado con esto, otro problema del enfoque mecanicista es el la subdivisión de las actividades. Los niveles de mecanismos parecen afectar solo a las entidades, de forma que las actividades, a pesar de tener para los autores adecuación óntica, parecen flotar entre niveles sin ser descomponibles en ninguno de ellos.

Asimismo, la asunción de adecuación óntica de las entidades también puede cuestionarse, pues solo el nivel más fundamental parece independiente de los intereses investigadores del observador. Por esta razón consideramos más adecuado hablar de entidades-sistema. Cada una de estas entidades-sistema interacciona con otras del mismo nivel en base a sus propiedades de nivel configurando un mecanosistema que visto desde la perspectiva superior supone una entidad-sistema del siguiente nivel.

Una posible crítica a este enfoque es la que presupone la necesidad de un análisis top-down previo para identificar las funciones. MCD (Machamer et al. 2000, 4) asumen esto, de forma similar a como hicieran los emergentistas británicos Samuel Alexander (Alexander 1920, 328) o C. Lloyd Morgan (Morgan 1923, 5). Esto supone conocer mediante la experiencia previa qué acciones llevan a cabo unas entidades concretas, en lugar de deducir las actividades a partir de las propiedades. Sin embargo, esta es una cuestión derivada principalmente del foco de interés del investigador. Al estudiar un aspecto fisiológico determinado, lo primero que hacemos es definir el objeto de estudio para posteriormente investigar hacia abajo. Pero no es por la imposibilidad de llegar a una conclusión escalando niveles, sino porque buscamos explicar algo relevante para nuestro interés. Esta relevancia a priori es también clave al considerar principales o secundarias las funciones de un mismo órgano. Pensemos, por ejemplo, en un músculo. A nivel molecular, por no profundizar más, la interacción entre actina, miosina, titina y demás, como proteínas estructurales del sarcómero, y de las enzimas involucradas, como las ATPasas, dan lugar, desde una perspectiva superior, a un acortamiento del sarcómero, pero también a una producción de calor. Es más, ni siquiera la generación de calor del músculo es una actividad única, sino la suma de los calores de reposo, de activación, de acortamiento, de recuperación y de relajación, producidos en diferentes reacciones, como la actividad de la bomba $\mathrm{Na}-\mathrm{K}$, la liberación de calcio, etc. (Michael y Sircar 2012, 84). La misma actividad molecular que produce la contracción conlleva el calor muscular. Solo el enfoque a priori determina la relevancia, y es la razón de la necesidad del análisis top-down previo. 


\subsection{INTERACCIÓN ENTRE ELEMENTOS DE DIFERENTE TAMAÑo}

Un aspecto interesante derivado de la división de la materia mediante ICDs es la imposibilidad de interacción entre entidades de diferente nivel. Desde esta perspectiva, la interacción entre entidades-sistema se hace exclusivamente en el nivel fundamental. Aunque resulte muy intuitivo decir que, en la sinapsis neuronal, el $\mathrm{Ca}^{2+}$ interacciona con la calmodulina, podemos observar como lo hace solo con ciertos componentes de ésta, pasando a formar parte de una nueva entidad-sistema, el complejo calcio-calmodulina, composicionalmente novedoso y estructuralmente diferente.

En uno de sus ejemplos más llamativos, Craver explicaba el proceso según el cual un virus entra en contacto con nada menos que un general del ejército (Craver y Bechtel 2006, 556). Es cierto que el ejemplo de Craver sigue una forma de hablar común y entendible. Parece evidente que cuando alguien dice algo como «Obama está manteniendo conversaciones con Cuba» puede entenderse que en realidad quiere decir «Obama ha conversado con Raúl Castro». Sin embargo, esta forma de hablar puede encubrir los procesos causales que ocurren realmente. El virus no interactúa con el general, del mismo modo que Obama no interactúa con Cuba. En realidad, son los átomos de las proteínas superficiales del virus los que entran en contacto con los átomos del glucocalix de las membranas de las células superficiales de la persona. La descripción de los casos biológicos que se suele hacer desde el mecanicismo deja ver un problema respecto a los criterios para decidir qué elementos forman un sistema y cuáles están fuera de él formando un entorno.

Por ejemplo, cuando el calcio está separado de la calmodulina resulta evidente que desde la perspectiva molecular no forman parte del mismo sistema. Pero, una vez unidos, y a pesar de no ser una unión covalente, la formación de un complejo de coordinación es claramente más fuerte que los enlaces débiles habituales, por lo que se podría considerar un nivel intermedio. Además, en estado de reposo, existe una alta concentración de complejo calciocalmodulina (Latorre 1996, 156) por lo que no está nada claro que se pueda hablar del calcio como extrasistémico. Del mismo modo, la distinción que hace Craver entre el corazón y un virus como intrasistémico y extrasistémico a la hora de hablar de la causa de la muerte de una persona se difumina cuando consideramos la forma de actuar de algunos virus. El caso del virus del herpes simple es aclarador. Tras una primera infección, el ADN viral permanece en el núcleo de las neuronas afectadas reactivándose a lo largo de los años. Otros virus se integran en el ADN del hospedador dividiéndose con él y, en el caso de darse en células de la línea germinal, pudiéndose transmitir a la descendencia en forma de elemento viral endógeno para reactivarse después. En ambos casos, hablar de intra y extrasistémico es claramente arbitrario.

\subsection{Causación internivel}

El enfoque manipulacionista de Craver es perfectamente entendible desde su perspectiva explicativa, no obstante, desde el enfoque aquí presentado, necesita algún comentario.

La visión salmoniana de líneas de mundo con un valor no nulo de una cantidad conservada y de la intersección de éstas formando redes de mundo es perfectamente válida siempre y cuando nos movamos en el nivel fundamental. Pero las simplificaciones hechas al determinar sistemas en los diferentes niveles introducen errores acumulados que dificultan la detección de los nodos. Estos cruces dejan de ser un punto para ser un conjunto de ellos tratado como si lo fueran. Cuanto más nos alejemos, más difusas serán las líneas y los nodos. 
Resulta evidente que la idea de interacción etiológica ideal y de manipulacionismo son tremendamente prácticas cuando se trata de establecer la causalidad de un fenómeno concreto, generalmente una función. El problema es que puede limitar la investigación al basarse en una decisión a priori. Sin este aspecto teleológico, la red de líneas de mundo previa a un momento no tiene nexos más relevantes que otros. Esta visión de la red causal sin finalidad explicativa concreta puede ayudar a descubrir relaciones de causalidad que pasarían desapercibidas en el caso de estar previamente dirigidas.

Sin embargo, una vez focalizada la atención causal en un evento E concreto ocurrido en un momento $t_{0}$, las redes de mundo precedentes que influyen en dicho evento están más restringidas cuanto más cerca de $t_{0}$ estemos. Así, en $t_{-1}$ habrá ciertos nodos que no puedan afectar a $E$ porque el tiempo en el que tardarían en influir es superior al diferencial de tiempo $d t$. De esta forma, conforme nos movamos hacia el pasado los nodos causales influyentes serán cada vez más numerosos. Esto determinaría un cono de nexos causales previos al evento, cada uno de los cuales está a su vez influido por los nodos anteriores, por lo que tendrían su propio cono previo. Este cono causal estaría delimitado una línea de horizonte entre causas influyentes y no influyentes en un evento concreto. De igual forma, los eventos futuros en los que influirá causalmente $\mathrm{E}$ formarán un cono causal futuro (ilustración 1$)$.

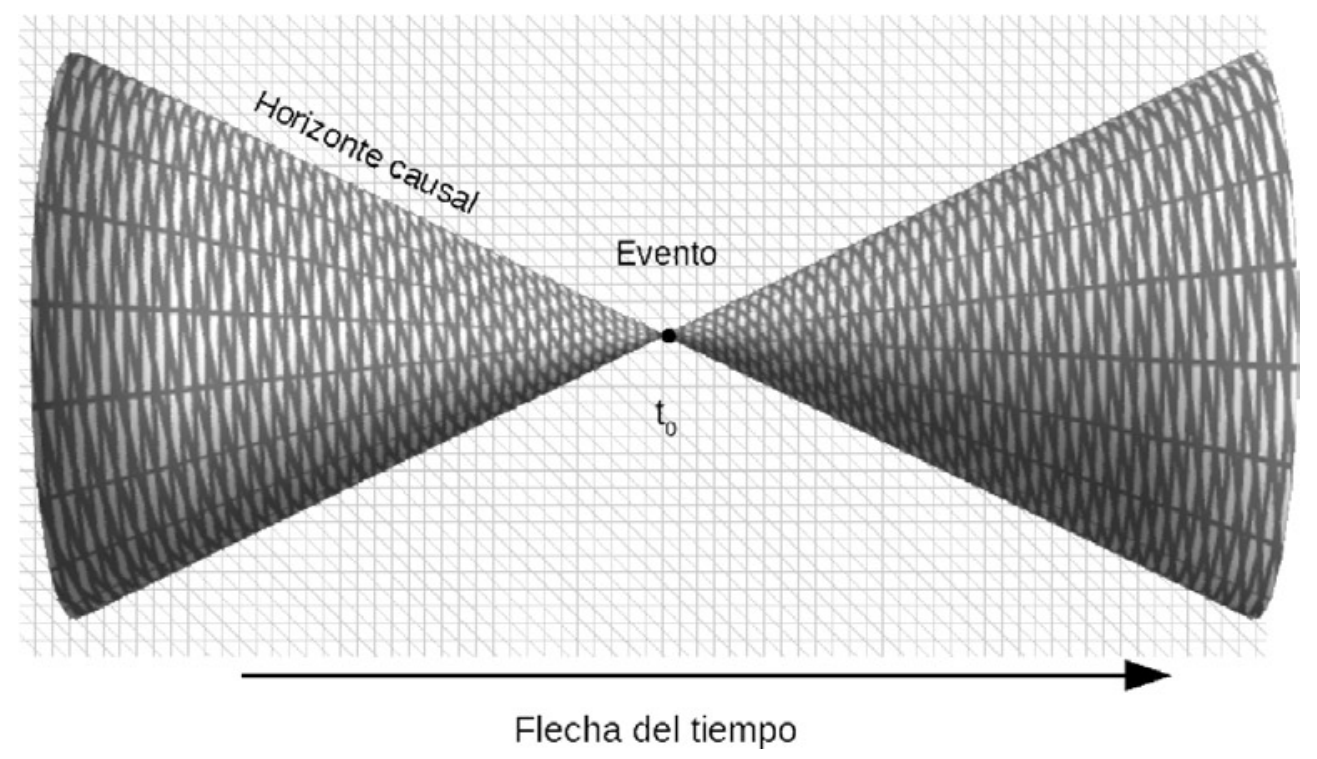

Ilustración 1. Cono causal

Por otro lado, el tratamiento dado a la causación internivel sin causas internivélicas es un intento de explicar la intuición de la investigación científica con un éxito cuestionable. Al investigar un fenómeno concreto, cierto número de nodos anteriores a su realización son lo que denominamos causas. Observando el nivel fundamental, la situación $F_{0}$ estará causada por otra previa $F_{-1}$, pero al ascender el nivel de foco, agruparemos cierta cantidad de ele- 
mentos del nivel $F$, por lo que a nivel macroscópico veremos que la situación $M_{0}$ está causada por una previa $M_{-1}$. Esta idea vale para cualquier nivel de observación:

Si observamos la causalidad de un acontecimiento A desde dos niveles distintos, y estamos interesados en la relación intranivel, es comprensible que analicemos aspectos diacrónicos como si fueran sincrónicos. Supongamos que queremos analizar la causa de $M_{0}$ en un nivel inferior, como quiera que esta situación es idéntica a $F_{0}$, su verdadera causa es la situación $F_{-1}$ que se corresponde con $M_{-1}$. Pero al no considerar el $d t$ transcurrido es fácil mezclar la explicación causal y la relación de identidad internivel y observar que $F_{-1}$ causa aparentemente $M_{0}$. Igualmente se puede tener la sensación de que $M_{0}$ mantenga una relación de condicionante sobre si misma visualizada como nivel microscópico, sin embargo, por la misma razón anterior, esto solo podría entenderse aparentemente sobre una situación micro posterior, y por lo tanto existir una causación descendente aparente entre $M_{0}$ y $F_{+1}$. (Cáceres y Saborido 2017, 103)

\subsection{UN EJEMPLO DE RELACIÓN ENTRE MECANOSISTEMAS: LA BACTERIA Y EL CORONEL}

En función de todo lo expuesto hasta ahora faltaría definir con la mayor claridad posible que es lo que entendemos por mecanosistema. Una primera aproximación podría ser la siguiente:

Un mecanosistema (MS) es un conjunto de entidades-sistema (E-S) pertenecientes a un intervalo de cuasi-descomponibilidad (ICD) cuya organización y propiedades $(\mathrm{P})$ son debidas exclusivamente a las propiedades de sus componentes. Donde:

— el ICD es elegido en función del interés del observador

- una entidad-sistema es a su vez un mecanosistema de un ICD inferior

— P son propiedades de nivel seleccionadas del ICD anterior.

Así, un mecanosistema quedaría definido por su ICD, sus entidades-sistema y sus propiedades:

$$
M S=d f(I C D, E-S, P)
$$

Faltaría por definir el criterio para la elección de un mecanosistema dentro del continuo de materia. Establecidos los niveles restaría definir cuáles de todas las E-S existentes en ese plano son las que van a formar parte del mecanosistema y por lo tanto que propiedades serán las que lo describan. Aquí se abre una doble posibilidad: a) se eligen los elementos y se observan las propiedades o b) se eligen las propiedades y se observan los componentes. De nuevo, la decisión entre las dos opciones es puramente heurística, siendo en general la opción b) la más utilizada en la ciencia: tras la observación de una propiedad se indaga en los elementos subyacentes en busca de aquellos responsables de ella. La búsqueda recursiva en niveles inferiores va construyendo un conjunto anidado de mecanosistemas formados por aquellos elementos que determinan la propiedad buscada. Sin embargo, este análisis $\mathrm{P} \rightarrow \mathrm{E}-\mathrm{S}$ tiene el inconveniente de dejar elementos fuera del mecanosistema no solo debido a la ignorancia o a los errores, sino en consideración de descomponibilidad de sistemas cuasi-descomponibles. Un estudio complementario E-S $\rightarrow \mathrm{P}$ puede ayudar a comprender mejor el funcionamiento del MS y a afinar mejor la definición de las propiedades. 
Haciendo una analogía con el citado ejemplo de Craver sobre el virus y la muerte del general (Craver y Bechtel 2006, 556), vamos a exponer en detalle un caso en que la bacteria Clostridium tetani mata a, digamos, un corone ${ }^{20}$. En este caso parece indiscutible que ambos eventos pueden ser vistos como causa y efecto. De hecho, si encontramos la forma de evitar ese contacto eliminando los reservorios bacterianos, se evitaría el tétanos. Pero si vamos más allá y queremos establecer con precisión las relaciones de causalidad y analizamos la sintomatología del tétanos, vemos que destaca la existencia de hipertonía, trismus, opistótonos y espasmos (Ardila et al. 2005, 174), todos ellos relacionados con una contracción continua de la musculatura estriada. Por lo tanto, por debajo del ICD de organismo (el propio coronel), el problema nos permite escoger las entidades-sistema implicadas, en este caso la musculatura estriada y su inervación. Al resto de elementos los eliminamos del mecanosistema a pesar de ser solo cuasi-descomponibles y por tanto poco influyentes.

Se denomina principio de inervación reciproca al hecho de que sean las mismas conexiones nerviosas las que inervan músculos agonistas y antagonistas, con la diferencia que la misma orden nerviosa que activa a los primeros, inhibe a los segundos. Esto implica simul-

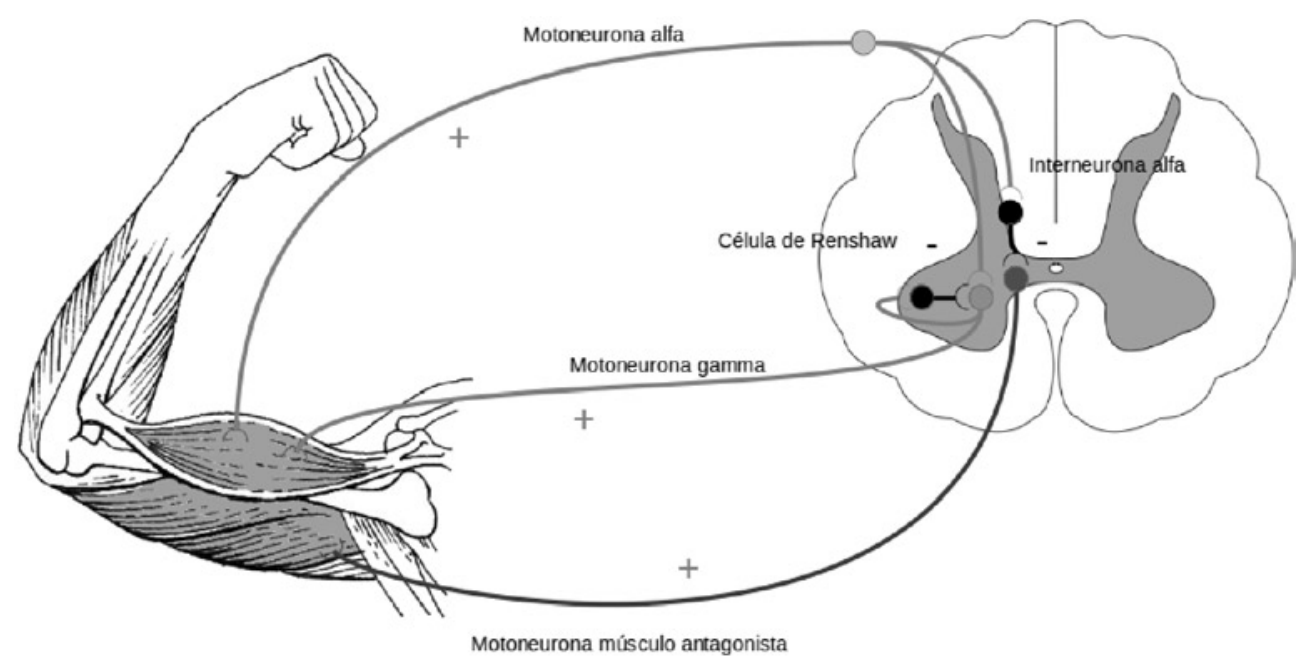

Ilustración 2

${ }^{20}$ Sostener que hay una relación causal más o menos directa entre microorganismos infecciosos y macroorganismos que enferman es algo habitual en la forma de entender la acción de virus o bacterias, y está presente ya en los postulados de Koch (Koch 1891). Así, los microorganismos que nos presentan estos postulados son agentes casuales que es posible identificar y aislar y que actúan en relación con otra entidad de nivel superior que podemos también identificar y aislar: el organismo (Gillies 2016). No es extraño que el ejemplo que Craver escoge para hablar de la causación internivélica sea uno en el que una pequeña entidad, un virus, es responsable de enfermar a otra de un tamaño mucho mayor (ver sección 4.3). Sin embargo, y aún a pesar de lo intuitivo del ejemplo, decir que una infección viral es un caso de causación internivel no sería más que el resultado de una simplificación de los procesos que realmente ocurren en una enfermedad infecciosa, simplificación que es denunciada frecuentemente en las revisiones críticas que se han planteado a los postulados Koch (p. ej., Fredericks y Relman 1996), (Gradmann 2014). 
taneidad en la contracción-relajación de grupos musculares contrarios evitando la contracción sincrónica y permitiendo, por ejemplo, el movimiento de elevación del antebrazo respecto del brazo.

Dentro del mecanosistema anterior, el problema está relacionado con la activación-inhibición de la contracción muscular, por lo que de todas sus E-S el estudio se va a centrar en la comunicación neuronal, no tomando en consideración el funcionamiento del músculo ni otros elementos componentes del mecanosistema. En este fenómeno intervienen las neuronas que inervan los músculos agonistas, motoneuronas alfa y gamma, sus equivalentes del músculo antagonista y dos interneuronas espinales, las neuronas Ia y las células de Renshaw. De todas ellas, las dos últimas son las encargadas de la inhibición, característica que hemos tomado como clave en la elección de nuestros mecanosistemas. Así, instalados en el nivel celular y basándonos en la elección de una propiedad, elegimos a las neuronas inhibidoras como nuevo mecanosistema.

Cuando las motoneuronas alfa y gamma reciben la orden de contraer un músculo, lo hacen mediante una sinapsis activadora mediada por acetilcolina. A su vez, la motoneurona alfa establece sinapsis activadora con la interneurona Ia y la motoneurona gamma hace lo propio con la célula de Renshaw. La primera, la neurona Ia, hace sinapsis inhibitoria mediada por GABA con las motoneuronas de los músculos antagonistas, mientras que la segunda, la célula de Renshaw, hace lo propio con la motoneurona gamma, en esta ocasión mediante el neurotransmisor inhibidor glicina (Ardila et al. 2005). Recordemos que el problema se debe a que los músculos que no deberían contraerse lo hacen debido a las neuronas inhibidoras. Así, éstas se constituyen como el nuevo mecanosistema, eliminando a las activadoras del análisis. Dentro de este mecanosistema nos centramos en la exocitosis de las vesículas cargadas de neurotransmisores inhibidores, tomando como componentes de nuestro mecanosistema solo aquellos elementos implicados.

Ya a nivel macromolecular, en la parte terminal de la neurona, ciertas proteínas transmembranales de las vesículas cargadas de neurotransmisores se encuentran unidas a moléculas de actina del citoesqueleto del axón. La acción de la proteína quinasa-II dependiente del complejo calcio-calmodulina, actuá sobre la sinapsina-I, una de las proteínas membranales de la vesícula, deshaciendo esta unión. Una vez libre en el citosol, la vesícula se desplaza hacia la membrana pre-sináptica. Asociada a la sinapsina-I y a la membrana vesicular se localizan también otras proteínas, entre las que destacan las sinapsinas-II y III, la sinaptobrevina, la sinaptotagmina y la sinaptofisina, todas ellas implicadas en la fusión de la membrana vesicular con la presináptica. Al aproximarse la vesícula a la membrana, la sinaptobrevina forma el complejo SNARE con las proteínas presinápticas, especialmente con la sintaxina y la SNAP-25, unión dependiente de calcio-calmodulina, que provocará la fusión de los lípidos de membrana de la vesícula con los de la membrana presináptica, permitiendo la liberación de los neurotransmisores a la brecha sináptica (Hu et al. 2003). Los distintos neurotransmisores llevan a cabo diferentes acciones, en función también del receptor postsináptico. Si su unión al receptor provoca la transmisión del impulso nervioso a la neurona siguiente, son activadores (acetilcolina, histamina), si no lo hacen, son inhibidores (glicina, GABA, serotonina).

La tetanoespasmina o toxina tetánica $(\mathrm{TeTN})$ es un péptido producido por las esporas vegetativas de la bacteria Clostridium tetani que entra en las neuronas en el lugar de la infección gracias a la acción de una de sus partes, la denominada cadena pesada. Una vez dentro, se desplaza vía retroaxonal hasta alcanzar, entre otras, a las neuronas inhibitorias espinales 
del SNC implicadas en la inhibición recíproca antagonista anteriormente citadas, la interneuronas Ia y las células de Renshaw. La cadena ligera tiene una acción endopeptidasa dependiente de cinc a través de la cual entra en contacto con la sinaptobrevina en la secuencia $\mathrm{Ala}(74)-\operatorname{Ser}(75)-\mathrm{Gln}(76)-\mathrm{Phe}(77)-\mathrm{Glu}(78)-\mathrm{Thr}(79)$. En presencia de cinc (Schiavo et al. 1992), la actividad endopeptidasa de la cadena ligera secciona la sinaptobrevina en la unión Gln76-Phe77, dando lugar a dos fragmentos aminoacídicos (Rood et al. 1997, 308). Esta fractura impide la formación del complejo SNARE, la fusión de las membranas y la liberación de neurotransmisores GABA y glicina. Escalando niveles, la no liberación de GABA y glicina, no da lugar a la inhibición de los músculos antagonistas, lo que da lugar a la contracción simultanea de ambos paquetes musculares dando lugar a una clínica que sin tratamiento concluye con la muerte del coronel (Ardila et al. 2005, 174).

Viendo la relación de causación dentro de cada nivel, observamos que a) la toxina rompe la sinaptobrevina, b) la neurona con toxina no propaga el impulso nervioso, c) el sistema nervioso intoxicado no regula la contracción muscular y d) la persona intoxicada acaba falleciendo. Si consideramos la sincronía que nos hace ver la causalidad real intranivélica como causalidad internivélica, diremos que la toxina causa la muerte, pues de hecho esto es lo que se percibe. De ahí la falsa apariencia de causación internivel, cuando, estrictamente hablando, la bacteria Clostridium tetani no mata al coronel, sino que interacciona con ciertas moléculas que conforman un subsistema del coronel y, una vez que lo hace, un componente de esta bacteria (la tetanoespasmina) forma ya parte del mismo sistema y se debe considerar como un componente más, de igual manera que ocurre cuando un ADN vírico se integra en los genomas de las células parasitadas en los ciclos lisogénicos.

\section{Conclusiones}

A largo de este trabajo, se han analizado los aspectos más significativos del denominado nuevo mecanicismo y hemos presentado una propuesta que trata de complementar esta perspectiva. Este enfoque introduce una razón para la compartimentación de la materia en niveles, la cuasi-descomponibilidad, que si bien permite elegir heurísticamente dichas divisiones, lo hace basándose en criterios que emanan de las propiedades de los elementos fundamentales de la materia. Creemos que puede afirmarse que estos criterios favorecen una elección de niveles arbitraria pero no caprichosa, lo que permite mantener una visión de la naturaleza como un continuo con la visión científica tradicional de que es posible distinguir niveles anidados. Además, lo hace sin tener que recurrir a propiedades emergentes como complemento a la explicación mecanística.

Considerar que los niveles explicativos se corresponden con los intervalos de cuasi-descomponibilidad permite elaborar una perspectiva sistémica de la explicación mecanística que propone la idea de mecanosistema como elemento central de la misma. La caracterización de los sistemas como entidades con mecanosistemas anidados conlleva una nueva interpretación de la causalidad inter e intranivélica y de la relación inter e intrasistémica, tal y como queda de manifiesto cuando observamos con detalle la relación entre la bacteria y el coronel. 


\section{REFERENCIAS}

Alexander, Samuel. 1920. Space, Time, and Deity. Vol 2. London: Mcmillan.

Ardila, Gustavo Pradilla, Jaime Otoniel Ayala Pimentel, y Claudia Liliana Sanabria Peña. 2005. Aprendizaje basado en problemas: Tétanos. Salud UIS 37 (3).

Barberis, Sergio. 2012. Un análisis crítico de la concepción mecanicista de la explicación. Revista Latinoamericana de Filosofia Vol. XXXVIII (2).

Bechtel, William, y Adele Abrahamsen. 2005. Explanation: A mechanist alternative. Studies in History and Philosophy of Science Part C: Studies in History and Philosophy of Biological and Biomedical Sciences 36 (2):421-441.

Bechtel, William, y Robert C Richardson. 1993. Discovering complexity: Decomposition and localization as scientific research strategies. Princeton, NJ: Princeton University Press.

Cáceres Vázquez, Emilio, y Cristian Saborido. 2017. Intervalos de cuasi-descomponibilidad y propiedades emergentes. Theoria 32 (1):89-108.

Cartwright, Nancy. 1994. Nature's Capacities and their Measurement. Clarendon Press. Oxford.

Churchland, Patricia S., y Terrence S. Sejnowski. 1988. Perspectives on cognitive neuroscience. Science 242 (4879):741-745.

Craver, Carl F. 2001. Role functions, mechanisms, and hierarchy. Philosophy of science 68 (1):53-74.

-. 2006. When mechanistic models explain. Synthese 153:355-376.

-. 2007. Explaining the brain: Mechanisms and the mosaic unity of neuroscience. Oxford University Press.

-. 2013. Functions and mechanisms: A perspectivalist view. En Functions: Selection and mechanisms, 133158. Springer.

Craver, Carl F., y William Bechtel. 2006. Top-Down causation without top-down causes. Biology and Philosophy 22:547-63.

Dowe, Phil. 2000. Physical Causation. Cambridge: Cambridge University Press.

Dupré, John. 2013. Living Causes. En Aristotelian Society Supplementary Volume, 87:19-37. Wiley Online Library.

Fredericks, DN, y David A Relman. 1996. Sequence-based identification of microbial pathogens: a reconsideration of Koch's postulates. Clinical microbiology reviews 9 (1):18-33.

Garson, Justin. 2013. The Functional Sense of Mechanism. Philosophy of science 80 (3):317-333.

Gillies, Donald Angus. 2016. Establishing Causality in Medicine and Koch's Postulates. International Journal of History and Philosophy of Medicine 6: 10603.

Glennan, Stuart. 1996. Mechanisms and the Nature of Causation. Erkenntnis 44:49-71.

-. 2002. Rethinking mechanisms explanation. Philosophy of Science 69:S342-S353.

-. 2008. Mechanism. En The Routledge Companion to Philosophy of Science, editado por Stathis Psillos y Martin Curd, 376-84. London; New York: Routledge.

Gradmann, Christoph. 2014. A spirit of scientific rigour: Koch's postulates in twentieth-century medicine. Microbes and infection 16 (11):885-892.

Hedström, Peter, y Petri Ylikoski. 2010. Causal mechanisms in the social sciences. Annual review of sociology 36:49-67.

Hu, Chuan, Mahiuddin Ahmed, Thomas J Melia, Thomas H Söllner, Thomas Mayer, y James E Rothman. 2003. Fusion of cells by flipped SNAREs. Science 300 (5626):1745-1749.

Koch, Robert. 1891. Ueber bakteriologische Forschung. En Verhandlung des X Internationalen Medichinischen Congresses, Berlin, 1890. Ed: August Hirschwald, Berlin. pp: 1-35.

Kuhlmann, Meinard, y Stuart Glennan. 2014. On the relation between quantum mechanical and neo-mechanistic ontologies and explanatory strategies. European Journal for Philosophy of Science 4 (3):337-359.

Latorre, Ramón. 1996. Biofísica y fisiología celular. Ed. Universidad de Sevilla.

Levy, Arnon. 2013. Three Kinds of New Mechanism. Biology and Philosophy 28:99-114.

Machamer, Peter, Lindley Darden, y Carl F. Craver. 2000. Thinking About Mechanisms. Philosophy of Science 67 (1):1-25. 
Michael, Joel, y Sabyasachi Sircar. 2012. Fisiología humana. México: El Manual Moderno.

Morgan, C. Lloyd. 1923. Emergent evolution. Editado por Williams y Norgate. $2^{\circ}$ edition (1927). London.

Oppenheim, Paul, y Hilary Putnam. 1958. Unity of science as a working hypothesis. En Concepts, theories, and the mind-body problem, Minnesota Studies in the Philosophy of Science II, editado por Herbert Feigl, Michael Scriven, y Grover Maxwell, 3-36. Minneapolis: University of Minnesota Press.

Penrose, Roger. 1991. La nueva mente del emperador. Barcelona: Grijalbo.

Rescher, Nicholas. 1996. Process metaphysics: An introduction to process philosophy. Suny Press.

Rood, Julian I., Bruce A. McClane, J. Glenn Songer, y Richard W Titball. 1997. The clostridia: molecular biology and pathogenesis. Academic Press.

Salmon, Wesley C. 1994. Causality without counterfactuals. Philosophy of Science, 297-312.

Schiavo, G., B. Poulain, O. Rossetto, F. Benfenati, L. Tauc, y C. Montecucco. 1992. Tetanus toxin is a zinc protein and its inhibition of neurotransmitter release and protease activity depend on zinc. The EMBO journal $11(10): 3577$.

Simon, Herbert A. 1962. The Architecture of Complexity. Proceedings of the American Philosophical Society $106(6): 467-82$.

Steel, Daniel. 2007. Across the boundaries: Extrapolation in biology and social science. Oxford University Press.

Weiss, Paul A. 1969. The living system: determinism stratified. En Beyond Reductionism: New Perspectives in the Life Sciences, editado por Arthur Koestler y John R. Smythies, 3-55. London, Hutchinson.

Wimsatt, William C. 1976. Reductionism, Levels of Organization, and the Mind-Body Problem. En Consciousness and the Brain: A Scientific and Philosophical Inquiry, editado por Irwin Savodnik (eds.) Walter B. Weimer (auth.) Gordon G. Globus, Grover Maxwell, 1. a ed. Springer US.

—. 1997. Aggregativity: Reductive Heuristics for Finding Emergence. Philosophy of Science 64(2):S372-84.

Woodward, James. 2003. Making things happen: A theory of causal explanation. Oxford University Press.

Wright, Cory D. 2012. Mechanistic explanation without the ontic conception. European Journal for Philosophy of Science 2 (3):375-394.

Emilio Cáceres Vázquez. Es licenciado en Ciencias Biológicas por la Universidad Complutense de Madrid, en Ciencias Ambientales por la UNED y máster en Filosofía Teórica y Práctica por la UNED. Actualmente está elaborando su tesis doctoral sobre Propiedades Emergentes. Es autor de varios libros de texto y de ensayo y profesor de enseñanza secundaria y de formación profesional.

Dirección: Departamento de Lógica, Historia y Filosofía de la Ciencia, UNED, Senda del Rey 7, 28040 Madrid, Spain. E-mail: emiliocaceres@epifumi.com

Cristian Saborido. Profesor del Departamento de Lógica, Historia y Filosofía de la Ciencia de la UNED. Doctor en Filosofía por la Universidad del País Vasco/Euskal Herriko Unibertsitatea. Especializado en Filosofía de la Ciencia, Filosofía de la Biología, Filosofía de la Medicina y Bioética. Autor de varias publicaciones en estas áreas en diversas compilaciones y revistas internacionales de investigación.

Dirección: Departamento de Lógica, Historia y Filosofía de la Ciencia, UNED, Senda del Rey 7, 28040 Madrid, Spain. E-mail: cristian.saborido@fsof.uned.es 